\title{
Numerical and Experimental Investigation of a Novel Thermal Management System Test Bench
}

\author{
Qing-guo LUO a, Hong-tao YIN ${ }^{\mathrm{b}}$, Dong-ya SI and Xiao-yu SI \\ Academy of Armored Forces Engineering, Beijing 100072, China \\ alqg_zgy@163.com, byinht2006@126.com
}

\begin{abstract}
Keywords: Thermal management system, Test Bench, Double cooling loops, Heat dissipations ability. Abstract: Increasing power requirements of military armored vehicles and space constrains of powertrain cabin result in some thermal parts, over heat load and more parasitic losses. Therefore, more intelligent Thermal Management Systems (TMS) are implemented, which with more than one cooling loops. A alternative Thermal Management System Test Bench (TMSTB) which with two cooling loops and three independent heat sources, was presented in this work utilized to investigate these problems. Numerical model is built and validated with experimental tests excellently. Computational results are used to investigate/research the system level characteristics of each loops and their relationship and relative influence of various parameters. In particular, fan speed has a great effect on the temperature of both cooling circuits. However the pump speed and fan speed should math with each other well to improve the heat dissipation ability of the system. Furthermore, some parameters, such as fan speed, pump speed, working fluid and the relationship between different loops have a considerable effect on the heat dissipation ability and controlled performance.
\end{abstract}

\section{Introduction}

Military ground vehicles are designed equipment with increasing power and weight and small volume, emission power and auxiliary power both increased. Components in the containment and small powertrain cabin over heat load. So TMS is paid attention[1]. A TMS is utilized as a useful technology with the purpose of reducing auxiliary power consumption on one hands, and on the other hand enforcing various components work steadily in favorite temperature range to prolong their life span[2].

The essence of a TMS is managing and controlling the flow of heat transfer media through which to transfer and emission heat[3]. Therefore, the characteristics and control strategy of active components, such as pump, fan and thermostat, are widely invested. Hydraulic controlled valves and servo-motor driven smart valves are invested to instead of traditional wax-based thermostat to route coolant flow through or bypass the radiator. Electric pump and fan are used to instead of mechanical ones which are driven by engine crankshaft[4]. At the same time, a series of control strategies are proposed.

A Thermal Management System Test Bench (TMSTB) is a practicable and sensible way to research TMS technology. While the way based on the prototype of whole vehicle have the disadvantages lie in long period, high cost and less flexibility[5]. A Test Bench features a hydraulic-based radiator fan, hydraulic-based water pump and hydraulic valves in a cooling circuit is assembled in Clemson University, coolant of which can be heated utilizing high temperature steam or immersion heater[6].

In this study a novel TMSTB are presented which has been designed and fabricated to functionally emulate a military ground vehicle's TMS. The primary device components include a subsystem of operating condition simulation subsystem which consist of three independent heat sources and utility an electric Heat Conducting Oil (HCO) boiler as original heat sources, cooling air duct, High Temperature Loop (HTL) and Low Temperature Loop (LTL). Numerical models which are validated with experimental tests are proposed to invest the system level characteristics of each loop and their relationship. 


\section{Modeling of TMSTB}

The TMSTB consist of three subsystems: operating condition simulation subsystem, air duct and coolant circuit pipes (HTL and LTL) (refer to Fig.1).

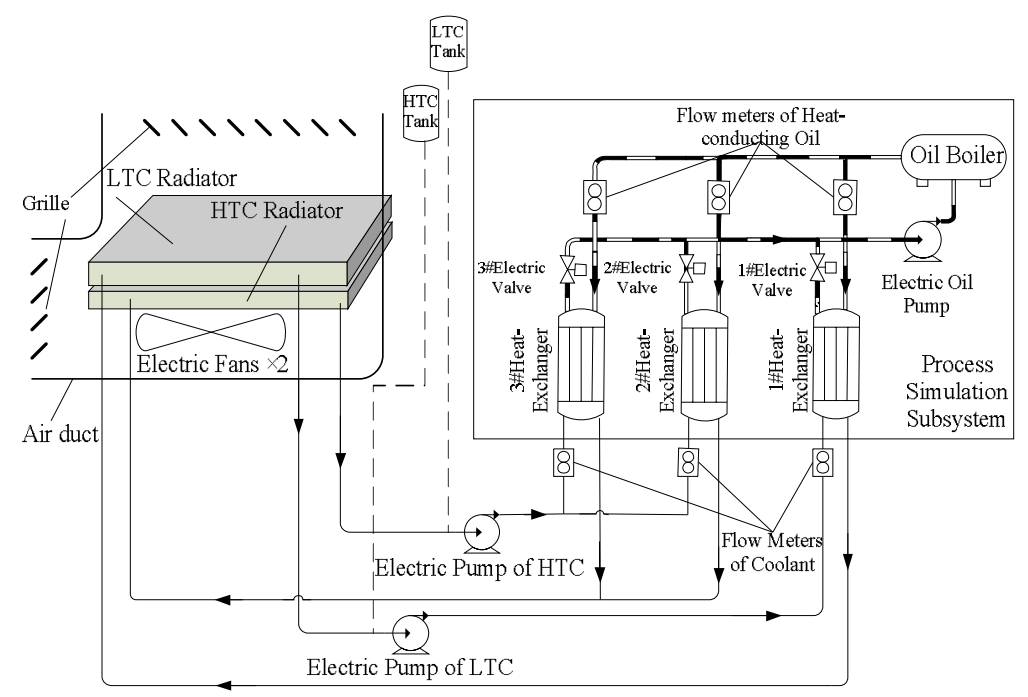

Fig. 1 Schematic of TMSTB

The operating condition simulation subsystem consists of an electric $\mathrm{HCO}$ boiler, three counter flow heat exchangers (HEX), servo-motor valves, flow meters and temperature sensors. The three servo-motor valves and the three flow meters are arranged at the inlet and outlet of high temperature side of HEX separately.

Models of Working state simulation subsystem, HTL and LTL, cooling air duct are present to invest characteristic of the TMSTB, predict the transient behavior and steady behavior of TMSTB.

Modeling of operating condition simulation subsystem. Lumped-parameter Models are used to describe the behavior of the operating condition simulation subsystem including HEX, servo-motor valve, Oil boiler and variable speed oil pump.

For the $i$ th HEX $(i=1,2,3)$, a thermal model with reduced order two nodes, high temperature oil and cooling coolant, and lumped parameter can be described by

$$
\begin{aligned}
& Q_{\text {in } \_i}(t)=q_{\text {oil_ } i}(t) c_{\text {oil }}\left(t_{\text {oil_in } \_i}-t_{\text {oil_out } \_i}\right) \\
& Q_{\mathrm{in} \_i}(t)=U A_{\mathrm{HEX}} \Delta T_{\mathrm{HEX} \_\mathrm{m}}(t) \\
& Q_{\text {out }}(t)=Q_{\text {in }}(t) \\
& =q_{\text {coolant } \_i}(t) \rho_{\text {coolant }} c_{\text {coolant }}\left(t_{\text {water_in_ } \_i}-t_{\text {water_out } \_i}\right) \\
& =q_{\text {oil_i } i}(t) \rho_{\text {oil }} c_{\text {oil }}\left(t_{\text {oil_in } \_i}-t_{\text {oil_out_ } i}\right)
\end{aligned}
$$

Where $Q_{\mathrm{in} \_i}$ denotes the input heat of cooling circuits through HEX, which are used to emulate the heat of various thermal parts in controllable and flexibly adjustable manner. The variable $q_{\mathrm{oil}_{\_} i}$ is the HTO mass flow rate at each HEX controlled by the opening of servo-motor valve and the speed of oil pump. $c_{\text {oil }}$ represents the specific heat of HTO. $t_{\text {oil_in } \_i}$ and $t_{\text {oil_out } \_i}$ denote the temperature of HTO at the inlet and outlet of the $i$ th HEX, the former of which is supported by Oil boiler and controlled by a PID controller.

$U$ and $A_{\mathrm{HEX}}$ is the equivalent heat transfer coefficient and equivalent heat transfer area of HEX, respectively. The signal $\Delta T_{\text {HEX_m }}(t)$ denotes the logarithmic mean temperature difference, which may be explicitly expressed as:

$$
\Delta T_{\mathrm{HEX} \_\mathrm{m}}=\frac{\Delta T_{\mathrm{I}}-\Delta T_{\Pi}}{\ln \left(\frac{\Delta T_{\mathrm{I}}}{\Delta T_{\Pi}}\right)}
$$


Where $\Delta T_{\mathrm{I}}=t_{\text {oil_in } \_i}-t_{\text {coolant_out } \_i}, \Delta T_{\Pi}=t_{\text {oil_out } \_i}-t_{\text {coolant_in } \_i} \cdot t_{\text {coolant_in } \_i}$ and $t_{\text {coolant_out_ } i}$ denote the temperature of coolant at the inlet and outlet of the $i$ th HEX.

The valves are driven by $12 \mathrm{~V}$ DC servo-motor, which integrated with external potentiometer for position feedback, attached to a screw connected to the valves' piston. The variable $H_{i}(t)$ denote the opening extent of the $i$ th valves satisfying $0 \leq H(t) \leq 1$. Note that $H(t)=1(0)$ represents a full opening (closing) condition. The relationship between pressure drop, $H_{i}(t)$ and flow rate $q_{\mathrm{oil}_{-} i}(t)$ is linear, which maybe expressed as:

$q_{\text {oil_ } i}(t)=K_{\mathrm{v}} q_{\max } \sqrt{\frac{\Delta p}{\Delta p_{\text {ref }}}} H(t)$

Where $q_{\max }$ is the maximum mass flow rate of HTF when the pressure drop across the valve is $\Delta p_{\text {ref }}$. $K_{\mathrm{v}}$ is a constant factor, which reflects the characteristics of the valve.

A performance data-based model was built.

$q_{\text {oil }}(t)=f\left(N_{\text {oil_pump }}(t), \Delta P_{\text {oil }}(t)\right)$

Where $N_{\text {oil_pump }}(t)$ denotes the variable pump speed. $q_{\text {oil }}(t)$ denotes the volume flow rate of the HTO.

$\Delta P_{\text {oil }}(t)=\Delta P_{\mathrm{HE}}(t)+\Delta P_{\text {oil_bypass }}(t)$

$\Delta P_{\text {oil_bypass }}(t)$ represent the resistance of HTF in the pipeline, can be calculated as:

$\Delta P_{\text {oil_bypass }}(t)=\frac{128 \mu L Q_{\text {oil }}(t)}{\pi d^{4}}$,

where $L$ and $d$ represent the length and diameter of oil pipe.

Let the local resistance coefficient of the three electric valve be $\xi_{o e x-1}, \xi_{o e x-2}, \xi_{o e x-3}$. Because the heat exchangers were disposed in parallel, so their pressure drop are equal:

$\frac{8 q_{v, o 1}^{2}}{\pi^{2} d_{2}^{4} g} \xi_{o e x-1}=\frac{8 q_{v, o 2}^{2}}{\pi^{2} d_{2}^{4} g} \xi_{\text {oex }-2}=\frac{8 q_{v, o 3}^{2}}{\pi^{2} d_{3}^{4} g} \xi_{\text {oex }-3}$

The overall flow rate of HTF pump is:

$Q_{o}=q_{v, o 1}+q_{v, o 2}+q_{v, o 3}$

The overall pressure drop is:

$H_{o}=\frac{\Delta p_{o}}{\rho_{o} g}+\frac{8 q_{v, o 1}^{2}}{\pi^{2} d_{2}{ }^{2} g} \xi_{o e x-1}$

Modeling of HTC and LTC. On the test bench, the temperature of coolant and HCO at inlets and outlets of heat exchanger could been measured by Temperature Sensors, so Heat Balance Equations were adopted to calculate the heat transfer capacity of heat exchanger.

$Q_{\mathrm{LTC}}(t)=U_{\text {LTC }} A_{\mathrm{LTC}} \Delta T_{\text {LTC_m }}(t)$

$Q_{\mathrm{HTC}}(t)=U_{\text {HTC }} A_{\text {HTC }} \Delta T_{\text {HTC_m }}(t)$

Where $t_{a 1}$ is the temperature of cooling air at the inlet, namely the temperature of ambient air, ${ }^{\circ} \mathrm{C} ; t_{a 2}$ is the temperature of the cooling air flowed through low-temperature radiator, ${ }^{\circ} \mathrm{C} ; t_{a 3}$ is the temperature of the cooling air flowed through high-temperature radiator, ${ }^{\circ} \mathrm{C} ; \Delta t_{a l}=t_{a 2}-t_{a 1}$, which is the temperature difference of cooling air between the inlet and outlet of low-temperature radiator, ${ }^{\circ} \mathrm{C} ; \Delta t_{a h}=t_{a 3}-t_{a 2}$, which is the temperature difference of cooling air between the inlet and outlet of high-temperature radiator.

High temperature circulation and low temperature circulation were respectively driven by high-temperature pump and low-temperature pump. Flow resistance of coolant can be expressed as: 
$\Delta P_{\text {coolant }}(t)=\frac{128 \mu L Q_{\mathrm{HTL}}(t)}{\pi d^{4}}$

The flow resistance of coolant consists of three parts flow resistance of radiator flow resistance of heat exchanger and on-way resistance of circulation pipeline, The Working characteristics of high-temperature pump and low-temperature pump were shown in Fig.2.

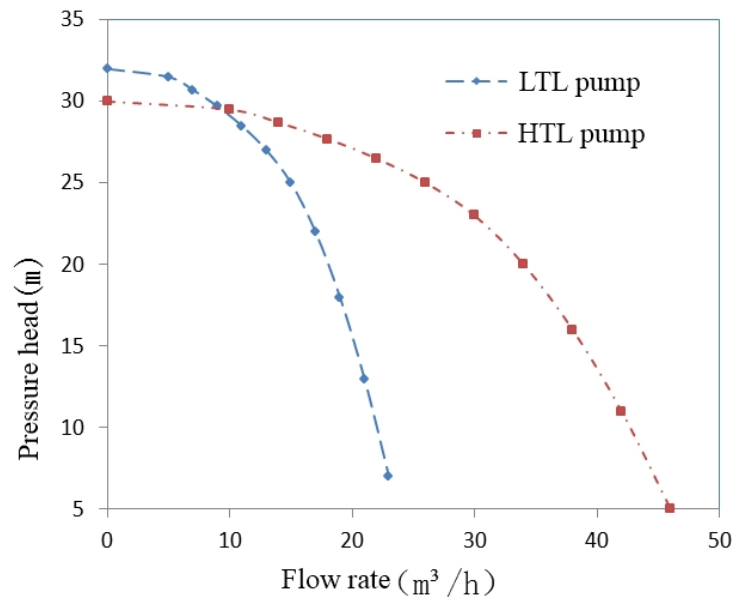

Fig.2 Working characteristics of high-temperature pump and low-temperature pump

According to the principle of similarity, when the speed of pump changed, the parameters should be recalculated by :

$\frac{n}{n_{m}}=\frac{Q}{Q_{m}}=\sqrt{\frac{H}{H_{m}}}=\sqrt{\frac{p}{p_{m}}}=\sqrt[3]{\frac{N}{N_{m}}}$

The on-way resistance of circulation pipeline is calculated by:

$\Delta p=\lambda \frac{L}{d} \times \frac{\rho v^{2}}{2}$

Where $L$ is the total length of circulation, $d$ is the dia of pipeline, $v$ is the velocity of coolant, $\rho$ is the density of coolant, $\lambda$ is the coefficient of the on-way resistance.

The flow resistance of radiators and heat-exchanger are equivalent to partial resistance, let the resistance coefficient of radiators and heat-exchangers on the coolant side be $\xi_{w r-i}$ and $\xi_{w e x-i}, \mathrm{i}=1,2,3$. Their resistance of flow are calculated by:

$$
\begin{array}{r}
\Delta p_{w r-i}=\xi_{w r-i} \times \frac{\rho_{w} v_{w r-i}{ }^{2}}{2} \\
\Delta p_{w e x-i}=\xi_{w e x-i} \times \frac{\rho_{w} v_{w e x-i}^{2}}{2}
\end{array}
$$

The total resistance of low-temperature circulation is calculated from the equation:

$\Delta p_{l}=\lambda_{w l} \frac{L_{l}}{d_{l}} \times \frac{\rho_{w} v_{l}^{2}}{2}+\Delta p_{w r-1}+\Delta p_{w e x-1}$

For the high-temperature circulation, because both radiators and heat-exchangers are in the circulation series connection, so the total flow resistance can be calculated as follows:

$\Delta p_{h}=\lambda_{w h} \frac{L_{h}}{d_{h}} \times \frac{\rho_{w} v_{h}^{2}}{2}+\Delta p_{w r-2}+\Delta p_{w e x-2}$

where $\Delta p_{w r-2}=\Delta p_{w r-3}, \Delta p_{w e x-2}=\Delta p_{w e x-3}$.

Modeling of air duct. Similarly, compared with real vehicle, the entire process space of cooling air flowed through, from inlet to the outlet, is called duct here. The duct was constituted mainly with intake duct segment, outtake duct segment, radiator, volute and grille. Centrifugal fan drive the cooling air flow in the duct, whose working character was showed in Fig.3. 


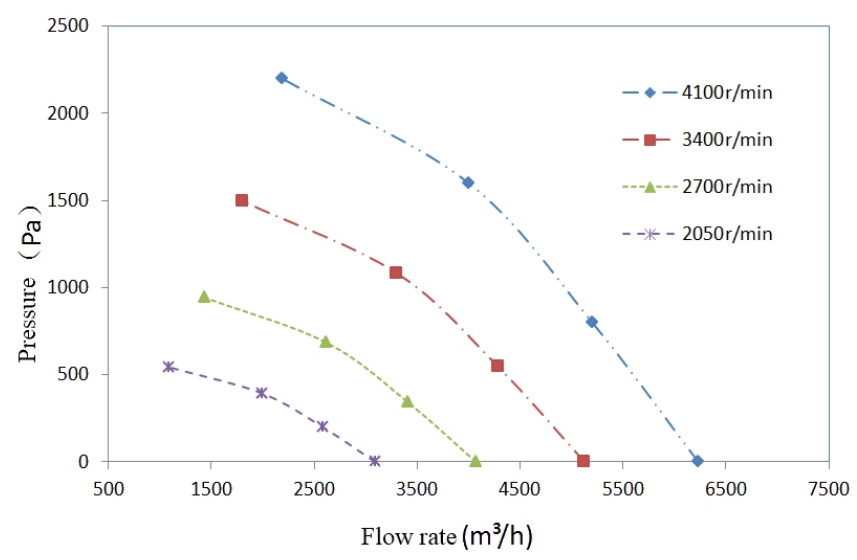

Fig. 3 Working characteristics of centrifugal fan

The duct resistance comes mainly from three parts: the radiators, air intake duct segment and air outtake duct segment.

$\Delta P_{\text {air }}=\Delta P+\Delta P+\Delta P+\Delta P$

The HTC radiator and LTC radiator share two electric centrifugal fans, the speed of which, $N_{\text {fan }}(t)$, impact the radiators' mass flow rate. The equation for the rotation speed of fan and the air speed, $V_{\text {air }}$, can be written as:

$\frac{d N_{\text {fan }}}{d t}=\frac{1}{J_{\text {fan }}}\left(-B_{\text {fan }} N_{\text {fan }}+T_{\text {fan }}-\rho_{\text {air }} A_{\text {fan }} R_{\text {fan }} V_{\text {air }}^{2}\right)$

Where $J_{\text {fan }}$ and $B_{\text {fan }}$ are the equivalent moment of inertia and viscous damping of the fan respectively. The cooling air mass flow rate can be determined as $m_{\text {air }}=\beta_{\text {rad }} V_{\text {air }} \rho_{\text {air }} A_{\text {fan }}$ where $A_{\text {fan }}$ is the fan face area.

The Local Resistance of radiator could be calculated as follows:

$\Delta p_{a 1}=\Delta p_{a l}+\Delta p_{a h}=\left(\xi_{a l}+\xi_{a h}\right) \times \frac{\rho_{a} \cdot v_{a}^{2}}{2}$

The Resistance Coefficient of the intake duct segment and outtake duct segment is respectively $\lambda_{\text {in }}$ and $\lambda_{\text {out }}$, which determined by the following equation:

$\Delta P=\frac{128 \mu L Q_{\text {air }}}{\pi d^{4}}$

$\Delta P=0.241 L \rho_{\text {air }}{ }^{0.75} \mu^{0.25} d^{-4.75} Q_{\text {air }}^{1.75}$

\section{Experiments}

The TMSTB was established /constructed, and a series of experiments including steady condition experiments and transient condition experiments were taken to validate the TMSTB's thermal behavior and fluid characteristics. The details are shown in Tab 1.

The test procedures involves setting heat exchangers power, $15 \mathrm{~kW}, 25 \mathrm{~kW}$ and $35 \mathrm{~kW}$ to simulate a heavy duty working conditions and a series of pump and fans' speed, which are shown in Tab.2 9groups test are tested. The HTL pump and LTL pump were with the same speed.

Tab. 1 The details of experiments

\begin{tabular}{|c|c|}
\hline Item & Description \\
\hline Working coolant & Water \\
\hline Ambient temperature & $27^{\circ} \mathrm{C}$ \\
\hline Ambient pressure & $890 \mathrm{kPa}$ \\
\hline
\end{tabular}


Tab.2 Groups of experiments

\begin{tabular}{|c|c|c|c|}
\hline \multirow{2}{*}{$\begin{array}{c}\text { Pump } \\
\text { speed/(r } \cdot \text { min-1 })\end{array}$} & \multicolumn{3}{|c|}{ Fan speed /(r $\cdot$ min-1) } \\
\cline { 2 - 4 } & 2000 & 2500 & 3000 \\
\hline 1500 & Case1 & Case2 & Case3 \\
\hline 2000 & Case4 & Case5 & Case6 \\
\hline 2500 & Case7 & Case8 & Case9 \\
\hline
\end{tabular}

Tab.3 Comparison of measured data and the model predictions

\begin{tabular}{|c|c|c|c|c|c|c|}
\hline \multirow[t]{2}{*}{ Case } & \multicolumn{3}{|c|}{ Temperature at $\mathrm{HTL} /{ }^{\circ} \mathrm{C}$} & \multicolumn{3}{|c|}{$\begin{array}{c}\text { Volume Flow rate of } \\
\text { coolant } / \mathrm{kg} / \mathrm{s}\end{array}$} \\
\hline & Measured & Predicted & Error & Measured & Predicted & Error \\
\hline 1 & 102.5 & 103.1 & $0.59 \%$ & \multirow{3}{*}{6.4} & \multirow{3}{*}{6.2} & \multirow{3}{*}{$\begin{array}{c}-3.13 \\
\%\end{array}$} \\
\hline 2 & 100.3 & 99.8 & $\begin{array}{r}-0.50 \\
\%\end{array}$ & & & \\
\hline 3 & 97.6 & 98.1 & $0.51 \%$ & & & \\
\hline 4 & 102.1 & 102.3 & $0.20 \%$ & \multirow{3}{*}{7.9} & \multirow{3}{*}{8.2} & \multirow{3}{*}{$3.80 \%$} \\
\hline 5 & 99.8 & 99.3 & $\begin{array}{r}-0.50 \\
\% \\
\end{array}$ & & & \\
\hline 6 & 96.7 & 97.1 & $0.41 \%$ & & & \\
\hline 7 & 101.5 & 100.9 & $\begin{array}{r}-0.59 \\
\%\end{array}$ & \multirow{3}{*}{10.6} & \multirow{3}{*}{11.8} & \multirow{3}{*}{$4.72 \%$} \\
\hline 8 & 96.8 & 96.1 & $\begin{array}{r}-0.72 \\
\%\end{array}$ & & & \\
\hline 9 & 84.3 & 82.3 & $\begin{array}{r}-2.37 \\
\%\end{array}$ & & & \\
\hline
\end{tabular}

The temperature at the inlet of radiator of LTL, temperature at the outlet of radiator of HTL and the volume flow rate of coolant are compared, between simulation and experiments, shown in Tab. 2 and Tab.3, where very good agreement is evidenced. The ability of the model to predict the operating characteristic during transient process was validated.

\section{Conclusions}

An experimental test bench named Thermal management system test bench(TMSTB) with HTL and LTL was introduced with the aim to research the technology of thermal management. A mathematical model of the TMSTB was established, and a experiments study were taken on the performance of the TMSTB. The TMSTB prototype were in excellent agreement with mathematical model which could be used to investigate characteristics of the TMS.

\section{Literature References}

[1] Cho, H., Jung, D., Filipi, Z., and Assanis, D., Application of Controllable Electric Coolant Pump for Fuel Economy and Cooling Performance Improvement, proceedings of the ASME IMECE, Advanced Energy Systems Division, vol. 44, pp. 43-50, Anaheim, CA, November 2004.

[2] Allen, D., and Lasecki, M., Thermal Management Evolution and Controlled Coolant Flow, SAE technical paper No. 2001-01-1732, 2001.

[3] Cho, H., Jung, D., Pilipi, Z., Assanis, D., Vanderslice, J., and Bryzik, W., 2007, Application of Controllable Electric Coolant Pump for Fuel Economy and Cooling Performance Improvement, Journal of Engineering for Gas Turbines and Power, 129, pp. 239-244.

[4] Chalgren, R., Jr., and Barron, L., Jr., 2003, Development and Verification of a Heavy Duty 42/14V Electric Powertrain Cooling System, SAE Paper No. 2003-01-3416. 
[5] Chalgren, R., Jr., 2004, Thermal Comfort and Engine Warm-Up Optimization of a Low-Flow Advanced Thermal Management System, SAE Paper No.2004-01-0047.

[6] Wagner J, Paradis I, Marotta E E, et al. Enhanced Automotive Engine Cooling Systems-A Mechahnnics Approach. International Journal of Vehicle Design, vo1 28, nos. 1/2/3, pp214-240, 2002 